\title{
Pseudoaneurysm of Internal Carotid Artery following Endoscopic Endonasal Pituitary Surgery Requiring Endovascular Treatment in an Acromegalic Patient with a Difficult Airway
}

\author{
Joanna S. Rodrigues ${ }^{1} \quad$ Ankit Gupta ${ }^{1} \quad$ Anil Parakh ${ }^{1}$ \\ ${ }^{1}$ Department of Anaesthesia, Global Hospitals, Mumbai, \\ Maharashtra, India
}

\begin{abstract}
Address for correspondence Joanna S. Rodrigues, MD Anesthesia, PDF Neuroanesthesia, A/11 Landmark, 4th Floor, CHS, Kadamwadi, Vakola, Santacruz (East), Mumbai 400055, Maharashtra, India (e-mail: joannarodrigues@yahoo.com).
\end{abstract}

\begin{abstract}
A pituitary tumor scheduled for surgical excision can eventuate with a myriad of challenges. A 49-year-old acromegalic patient was admitted to our hospital for trans-nasal trans-sphenoidal pituitary surgery. Magnetic resonance imaging (MRI) of his brain revealed a well-defined lesion involving right half of the anterior lobe of pituitary. In the operating room, we encountered difficulty in management of the airway. The trachea could be intubated using fiberoptic bronchoscope via a proseal laryngeal mask airway after five attempts of failed intubation. Intraoperatively, the right internal

Keywords

- airway management

- endotracheal intubation

- pituitary adenoma

- acromegaly

- pseudoaneurysm carotid artery (ICA) was injured during excision of lateral part of the tumor with a blood loss of 3.5 L. Adenosine use to aid hemostasis was unsuccessful. Endovascular coil embolization was done to treat the pseudoaneurysm developed in the right ICA, to restore the blood flow. During this process, the patient developed medial rectus palsy and ptosis in the right eye, which improved gradually. The above amalgamation of maladies commanded an integrated and well-coordinated team approach to manage the catastrophic complications.
\end{abstract}

\section{Introduction}

A pituitary disease is akin to a Pandora's box. Its multisystem essence poses a host of challenges to the anesthesiologist. Among them is the potential for difficult airway, which can be quite a daunting experience. Acromegaly is a functional pituitary tumor with excess growth hormone secretions leading to physical manifestations. The airway anatomy is altered due to thickening of the pharyngeal and laryngeal tissues, hypertrophy of the periepiglottic region, and calcinosis of the larynx leading to narrowing of the glottic ${ }^{1}$ opening, thus making it a difficult airway. The trans-sphenoidal surgery (TSS) for removal of pituitary tumor has been popularized due to its efficacy, safety, and a low margin of morbidity and mortality. However, the adaptation of transsphenoidal route as a surgical corridor is associated with grave complications such as vascular injury. ${ }^{2-4}$ Injury to the internal carotid artery (ICA) is the most feared complication, leading to catastrophic clinical deterioration of the patient.

In the event of unexpected massive bleeding during endonasal surgery, immediate control of hemorrhage is often resorted to by packing. ${ }^{5}$ Once hemostasis is achieved, computed tomography angiography (CTA) or digital subtraction angiography (DSA) can assist in identifying underlying arterial injury as a potential cause of hemorrhage. Traditionally, emergency surgical ligation was used to control the bleeding. Ironically, this treatment itself was ineffective and detrimental with complications resulting in stroke and death. ${ }^{6.7}$ Recent advances in endovascular techniques have, however, created alternatives to this traditionally high-risk technique. Pseudoaneurysms of the ICA have been reported to be associated with TSS. They lack a wall and should be treated as parent artery occlusion. ${ }^{8}$
License terms

() (1) $\ominus \circledast$ 
We report a case of ICA pseudoaneurysm developing after endoscopic endonasal surgery with safe and effective treatment with endovascular coils, thus allowing preservation of the parent artery and occlusion of the iatrogenic pseudo aneurysm in an acromegalic patient with an unanticipated difficulty in airway management with torrential hemorrhage intraoperatively.

\section{Case Report}

A 49-year-old man (weight, $79 \mathrm{~kg}$; height,175 cm) presented to our institution with complaints of headache, neck pain, and joint pain since the past 3 months. He was a diabetic for 2 years with features of acromegaly and no history of obstructive sleep apnea. Hormonal studies unveiled elevated growth hormone level of $55.60 \mathrm{ng} / \mathrm{mL}(0.05-8.0 \mathrm{ng} / \mathrm{mL})$, IGF-1 1,286 ng/mL (87-238 ng/mL), prolactin $5.03 \mathrm{ng} / \mathrm{mL}$ (2-15 ng/mL in males), 8 a.m serum cortisol level $3.80 \mathrm{ug} / \mathrm{dL}$ (5-23 $\mu \mathrm{g} / \mathrm{dL})$, serum testosterone $146.19 \mathrm{ng} / \mathrm{dL}$ (260-1,000 ng/dL), and free thyroxine (T4) $1.02 \mathrm{ng} / \mathrm{dL}$ (0.93-1.7 ng/dL). Magnetic resonance imaging (MRI) revealed a well-defined lesion involving the right half of the anterior lobe of pituitary gland with extension to the right cavernous sinus and suprasellar cistern (-Fig. 1). The rest of the laboratory results were normal. Echocardiography findings reported an ejection fraction of $60 \%$ with sclerotic mitral and aortic valve leaflets. On examination, the airway was Mallampati class II with a large and thick tongue. The patient was scheduled for a surgical decompression and excision of the tumor via trans-nasal trans-sphenoidal endoscopic approach. After an overnight period of fasting, the patient was shifted to the operating theatre (OT). In the OT, two $16 \mathrm{G}$ peripheral lines and an arterial line was placed in the left radial artery for realtime monitoring of blood pressure along with Lead II and V5 electrocardiogram and pulse oximeter. Difficult airway trolley with all the gadgets for difficult airway was kept ready including a cricothyrotomy and tracheostomy set. The presence of an otolaryngologist was sought during airway management. Intravenous (IV) $100 \mathrm{mg}$ of hydrocortisone was administered prophylactically. Anesthesia was induced with $100 \mu \mathrm{g}$ of IV fentanyl, $80 \mathrm{mg}$ of IV lignocaine, $150 \mathrm{mg}$ of IV propofol till loss of verbal response, and $8 \mathrm{mg}$ of IV vecuronium bromide

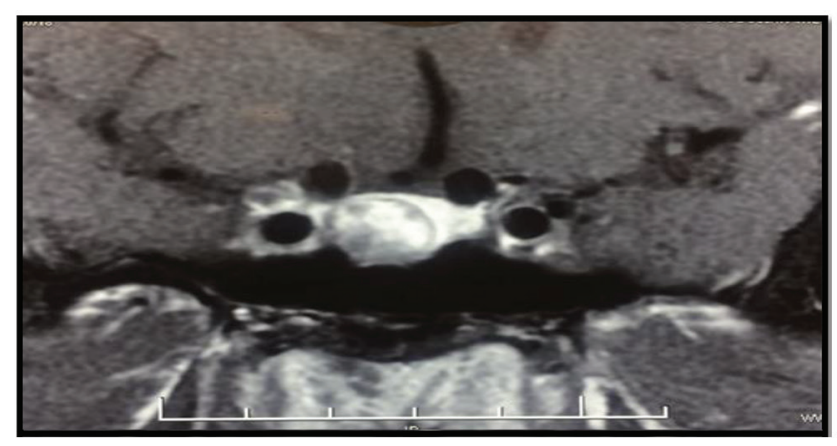

Fig. 1 Magnetic resonance imaging shows a uniformly enhancing sellar mass extending into the suprasellar cistern with mass effect on the optic chiasm. (after assessing bag and mask ventilation). We encountered three failed attempts to perform tracheal intubation using the Miller blade. The laryngoscopic view did not improve, even with adequate repositioning of the head with backward, upward, and right lateral displacement of the thyroid cartilage. After another course of bag mask ventilation, we attempted video laryngoscopy. However, due to the pronounced anterior anatomy of the larynx we faced difficulty maneuvering the styletted endotracheal tube (ETT) in the oropharynx. We failed to even guide the coude tip of the bougie via the vocal cords using video laryngoscopy. We attempted oral fiber optic intubation thrice using a 5.5-mm fiberscope. The first two attempts were unsuccessful due to bloody vision. On the third attempt, we were able to visualize the cords; however, we could not negotiate the tip of the fiber optic through the glottic chink. As oxygen saturation could be maintained at $95 \%$ using bag-valve-mask ventilation and the patient was not at an increased risk of aspiration of gastric contents, we decided to perform tracheal intubation via an appropriately sized laryngeal mask airway (LMA). We inserted a size 5 LMA easily that functioned properly. Later on, we passed a 6.5- mm ETT through the LMA with the aid of a fiber optic bronchoscope into the trachea. We then passed a tube exchanger with an outer diameter of $3.7 \mathrm{~mm}$ (Cook Critical Care; Bloomington, IN, United States) through the 6.5-mm ETT to exchange the 6.5-mm ETT and LMA with an 8-mm flexometallic tracheal tube. After successfully securing the airway, a throat pack was inserted and labeled. Anesthesia was maintained with desflurane along with oxygen and air. Patient was positioned supine with the upper torso and head elevated. During the surgery, we encountered a vascular injury incurring a blood loss of 3.5 L during excision of the lateral extension of the tumor which mandated us to transfuse five units of packed red blood cell. Despite providing tamponade with a variety of materials including Surgicel (original absorbable hemostat; Johnson \& Johnson; United States), muscle plugs, and tissue adhesives, the source of bleeding could not be identified and after arriving to a consensus with the surgeon, we decided to use adenosine $(0.3 \mathrm{mg} / \mathrm{kg})$ to provide for cardiac stand still and locate the point of bleeding. We administered about $48 \mathrm{mg}$ of adenosine in two doses. There was spontaneous recovery in the heart rate. Surgicel, gel foam, and nasal packs were used to stop the bleeding. The surgery was completed in 8.5 hours, and the patient was shifted for a CT scan which suggested pseudoaneurysm of the right carotid artery. It was decided to manage the iatrogenic pseudoaneurysm by endovascular approach using coil embolization (-Fig. 2). A total of 1,000 international units (IUs) of heparin was administered intravenously. Check angiogram revealed opacification of the pseudo aneurysm with a good flow in the right anterior cerebral artery and right middle cerebral artery through ICA and posterior circulation. Patient was shifted to intensive care unit (ICU), mechanically ventilated and trachea was extubated on the third postoperative day in OT, when he was taken up for nasal pack removal under general anesthesia. Patient developed medial rectus palsy and right eye ptosis which improved gradually till the date of discharge. 


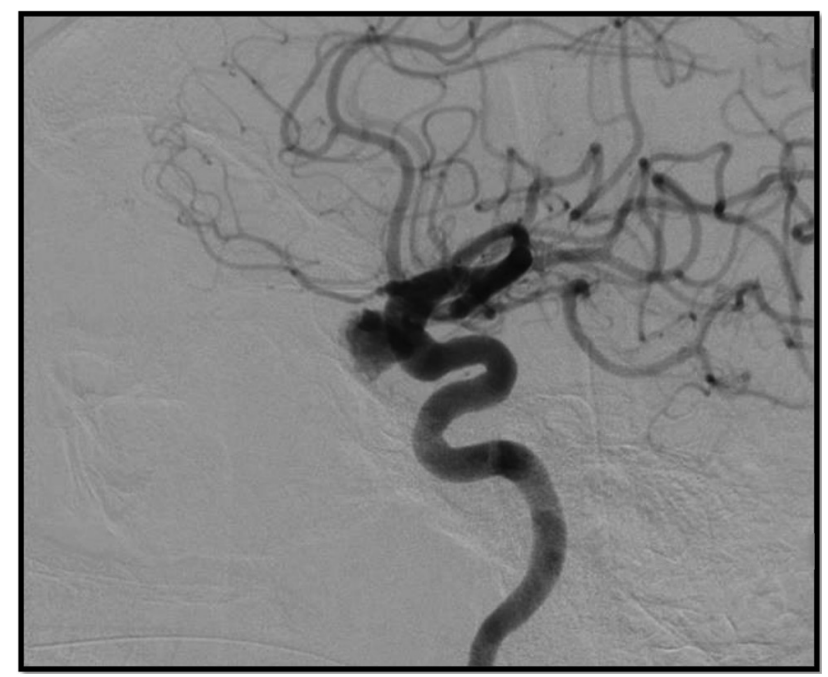

Fig. 2 Catheter angiogram showing and confirming the presence of the pseudoaneurysm.

\section{Discussion}

The perioperative anesthetic care of patients presenting for pituitary surgery requires careful preoperative assessment and meticulous pre- and postoperative management.

The incidence of difficult intubation in acromegalic patients is higher than the general surgical population. The unregulated hypersecretion of growth hormone in acromegalics results in several alterations in the airway anatomy. ${ }^{9}$ Patients develop hypertrophy of the facial bones and coarsening of features. The mandible becomes thicker and significant prognathism may be evident. Soft tissues of the nose, mouth, and tongue are equally affected. Significant macroglossia and hypertrophy of the laryngeal and pharyngeal soft tissues have also been well documented. ${ }^{10}$ The Mallampati classification has poor negative predictive value in acromegalics and difficult intubation may be unpredictable. The Mallampati score of our patient was II; hence, we chose to proceed without awake fiberoptic initially. However, we were ready with the difficult airway cart. Bindra et al ${ }^{11}$ conducted a study to compare extended Mallampati score (EMS) with modified Mallampati classification (MMP) in predicting difficult laryngoscopy in acromegalic patients. However, they did not find any additional benefit of neck extension while doing MMP for preoperative airway assessment. The apnea/hypoxia index and insulin-like growth factor I (IGF-I) levels are both increased in acromegalic patients with difficult intubation, and elevated IGF-I levels are an independent risk factor of difficult intubation in acromegalic patients. Our patient had excessively high levels of IGF-I, which could have provided us with a clue to the difficult intubation.

Out of the many complications associated with transsphenoidal surgery, vascular injury is the most dreaded. It occurs during aggressive resection of the tumor by damaging the surrounding vessels and sinuses. ${ }^{6}$ The most common causes of bleeding during transsphenoidal pituitary surgeries include arterial injuries to sphenopalatine artery (injured during sphenoidal opening) and ICA. Patients with acromegaly are

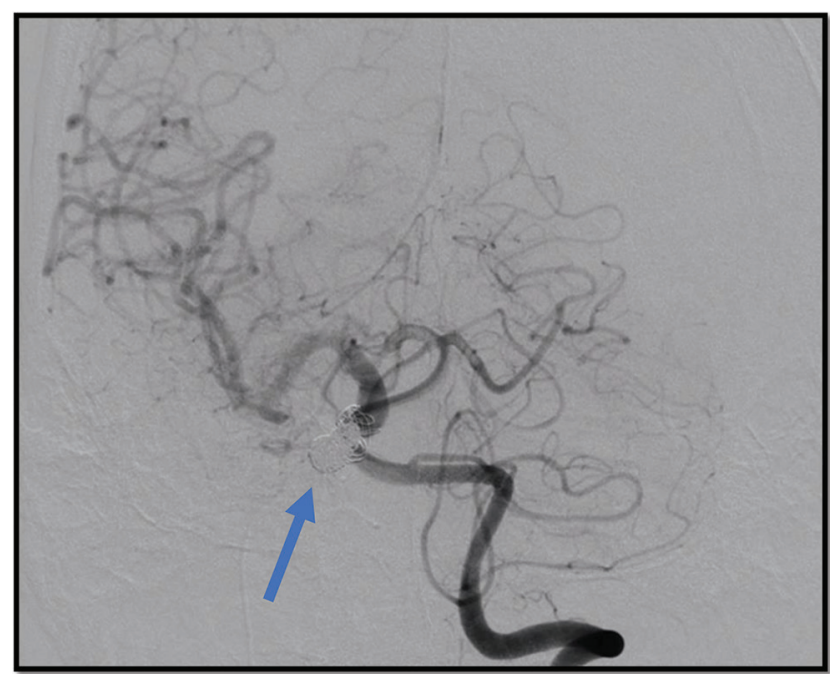

Fig. 3 Postcoil embolization angiogram of the right ICA shows successful occlusion of the pseudoaneurysm (arrow). ICA, internal carotid artery.

at increased risk of injury due to tortuous and ectatic carotid arteries that may protrude into the sella. ${ }^{12}$ Intraoperative ICA rupture creates an immediately challenging surgical field due to a high pressure/high flow hemorrhage scenario, which may rapidly result in exsanguination of the patient. We replenished the lost blood immediately and equalized the hemodynamic status. Packing materials ideally should be placed with just enough force to control the hemorrhage but not to occlude vascular flow. ${ }^{5}$ The vessel injury can also result in pseudoaneurysm formation, even though the artery appears to be structurally intact, packed, and not bleeding. To identify the source of bleeding, we used adenosine-induced systole. If excessive bleeding occurs during or after transsphenoidal surgery, even after successful intraoperative tamponade, immediate vascular imaging should be obtained to rule out vessel wall damage. ${ }^{13}$ If the neck is wide, the pseudoaneurysm can still be embolized with catheter-directed delivery of embolization materials. ${ }^{14}$ Due to the tortuous course of the vascular system, we could not navigate and negotiate the stent and hence resorted to coil embolization. A total of seven coils were employed for the same ( - Fig. $\mathbf{3}$ ).

What was unique about this case is that what seemed to be a straight forward excision of a pituitary adenoma proved to be otherwise, right from confronting us with an unanticipated difficulty in an anticipated difficult airway, intraoperative hemorrhage, and then coil embolization of the pseudo aneurysm. Though arduous, it was well managed by our team and here in resonates the words of Lailah Gifty Akita stating, "In the situation, we must adapt, survive, and strive."

\section{Conclusion}

The perioperative anesthetic care of patients presenting for pituitary surgery requires careful preoperative assessment and meticulous pre- and postoperative management. The presence of either an intraoperative suspicion of vascular injury or any neurologic symptoms and bleeding after transsphenoidal surgery must prompt one to perform rapid 
angiography to evaluate for vascular injury. An endovascular approach to the treatment of ICA pseudoaneurysm after transsphenoidal pituitary surgery may be an alternative method with equivalent effectiveness.

\section{Conflict of Interest}

None declared.

\section{References}

1 Nanjundaswamy NH, Sridhara RB. Perioperative management of patients with acromegaly-a retrospective analysis. International Journal of Research in Medical Sciences 2018;6(2):515-520

2 Berker M, Hazer DB, Yücel T, et al. Complications of endoscopic surgery of the pituitary adenomas: analysis of 570 patients and review of the literature. Pituitary 2012;15(3):288-300

3 Guiot G. Trans-sphenoidal approach in surgical treatment of pituitary adenomas: General principles and indications in non-functioning adenomas. In: Kohler PO, Ross GT, eds. Diagnosis and Treatment of Pituitary Adenomas. International Congress Series, No. 303. Amsterda Excerpta Medica;1973:159-178

4 Fatemi N, Dusick JR, de Paiva Neto MA, Kelly DF. The endonasal microscopic approach for pituitary adenomas and other parasellar tumors: a 10-year experience. Neurosurgery 2008;63(4, Suppl 2):244-256, discussion 256

5 AlQahtani A, Castelnuovo P, Nicolai P, Prevedello DM, Locatelli D, Carrau RL. Injury of the internal carotid artery during endoscopic skull base surgery: prevention and management protocol. Otolaryngol Clin North Am 2016;49(1):237-252

6 Raymond J, Hardy J, Czepko R, Roy D. Arterial injuries in transsphenoidal surgery for pituitary adenoma; the role of angiography and endovascular treatment. Am J Neuroradiol 1997;18(4):655-665

7 Chaloupka JC, Putman CM, Citardi MJ, Ross DA, Sasaki CT. Endovascular therapy for the carotid blowout syndrome in head and neck surgical patients: diagnostic and managerial considerations. Am J Neuroradiol 1996;17(5):843-852

8 Ahuja A, Guterman LR, Hopkins LN. Carotid cavernous fistula and false aneurysm of the cavernous carotid artery: complications of transsphenoidal surgery. Neurosurgery 1992;31(4):774-778, discussion 778-779

9 Dostalova S, Sonka K, Smahel Z, Weiss V, Marek J, Horinek D. Craniofacial abnormalities and their relevance for sleep apnoea syndrome aetiopathogenesis in acromegaly. Eur J Endocrinol 2001;144(5):491-497

10 Kitahata LM. Airway difficulties associated with anaesthesia in acromegaly. Three case reports. Br J Anaesth 1971;43(12):1187-1190

11 Bindra A, Prabhakar H, Bithal PK, Singh GP, Chowdhury T. Predicting difficult laryngoscopy in acromegalic patients undergoing surgery for excision of pituitary tumors: A comparison of extended Mallampati score with modified Mallampati classification. J Anaesthesiol Clin Pharmacol 2013;29(2):187-190

12 Hatam A, Greitz T. Ectasia of cerebral arteries in acromegaly Acta Radiol Diagn (Stockh) 1972;12(4):410-418

13 Karadag A, Kinali B, Ugur O, Oran I, Middlebrooks EH, Senoglu M. A case of Pseudoaneurysm of the internal carotid artery following endoscopic endonasal pituitary surgery: endovascular treatment with flow-diverting stent implantation. Acta Med (Hradec Kralove) 2017;60(2):89-92

14 Saad NE, Saad WE, Davies MG, Waldman DL, Fultz PJ, Rubens DJ. Pseudoaneurysms and the role of minimally invasive techniques in their management. Radiographics 2005;25(Suppl 1):S173-S189 